\title{
ON THE NUMBER OF APPARENT DOUBLE POINTS OF $r$-SPACE CURVES
}

BY B. C. WONG

Consider a curve $C^{N}$ of order $N$ in $r$-space. The number, $h$, of $(r-2)$-spaces passing through a given $(r-3)$-space and meeting $C^{N}$ twice is finite. If $C^{N}$ is projected on to a 3 -space, then $h$ is the number of apparent double points on the projection. To avoid circumlocution, we shall use the phrase the apparent double points of $C^{N}$ instead of the apparent double points of the 3-space projection of $C^{N}$.

When the curve $C^{N}$ is the intersection of $r-1$ hypersurfaces of order $n_{1}, n_{2}, \cdots, n_{r-1}$, the number of its apparent double points is known and is given by the formula*

$$
h=\frac{1}{2} n_{1} n_{2} \cdots n_{r-1}\left(n_{1} n_{2} \cdots n_{r-1}-\sum n_{i}+r-2\right) .
$$

But suppose $C^{N}$ is not the intersection of $r-1$ hypersurfaces but the intersection of $q<r-1$ varieties $V_{r_{1}}^{n_{1}}, V_{r_{2}}^{n_{2}}, \cdots, V_{r_{q}}^{n_{q}}$ of orders $n_{1}, n_{2}, \cdots, n_{q}$ and of dimensions (which may be different) $r_{1}, r_{2}, \cdots, r_{q}$ where

$$
r_{1}+r_{2}+\cdots+r_{q}=r(q-1)+1 .
$$

What is the formula for $h$ for such a curve? It is our purpose in this paper to derive this formula.

As a first step in the derivation, let $q=2$. Then $C^{N}$ or $C^{n_{1} n_{2}}$ is the intersection of two varieties $V_{r_{1}}^{n_{1}}, V_{r_{2}}^{n_{2}}$, where $r_{1}+r_{2}=r+1$. Let $h_{i}$ be the number of apparent double points on the curve $C^{n_{i}}$ in which an $S_{r_{i}}$ meets $V_{r_{i}}^{n_{i}}$. Decompose one of the given varieties, say $V_{r_{1}}^{n_{1}}$, into $n_{1} r_{1}$-spaces having severally $\frac{1}{2} n_{1}\left(n_{1}-1\right)-h_{1}$ $\left(r_{1}-1\right)$-spaces in common. The curve $C^{n_{1}}$ in which an $S_{r_{2}}$ meets the decomposed $V_{r_{1}}^{n_{1}}$ is, then, composed of $n_{1}$ lines forming a skew $n_{1}$-sided polygon with $\frac{1}{2} n_{1}\left(n_{1}-1\right)-h_{1}$ vertices. Now the curve $C^{n_{1} n_{2}}$ in which $V_{r_{2}}^{n_{2}}$ meets the decomposed $V_{r_{1}}^{n_{1}}$ is composed of $n_{1}$

* Veronese, Behandlung der projectivischen Verhältnisse der Räume von verschiedenen Dimensionen durch das Princip des Projicirens und Schneidens, Mathematische Annalen, vol. 19 (1882), pp. 161-234. The formula above is given on p. 205. 
curves all of order $n_{2}$. If any two of these $n_{1}$ curves intersect, they must intersect in $n_{2}$ points lying in one of the $\frac{1}{2} n_{1}\left(n_{1}-1\right)-h_{1}$ $(r-1)$-spaces mentioned above. Each of these $\left(r_{1}-1\right)$-spaces contains $n_{2}$ such points. Hence, the total number of points in which the $n_{1}$ curves actually intersect severally is seen to be $n_{2}\left[n_{1}\left(n_{1}-1\right) / 2-h_{1}\right]$. The total number of intersections, both actual and apparent, of the $n_{1}$ curves two by two is $\frac{1}{2} n_{1} n_{2}{ }^{2}\left(n_{1}-1\right)$. Now each of the $n_{1}$ curves has $h_{2}$ apparent double points. Therefore, we conclude that the number $h$ of apparent double points on the curve $C^{n_{1} n_{2}}$, proper or improper, is equal to the sum of the number of apparent intersections of the component curves of the degenerate $C^{n_{1} n_{2}}$ and the total number of the apparent double points on the component curves, that is,

$$
\begin{aligned}
h & =\frac{1}{2} n_{1} n_{2}^{2}\left(n_{1}-1\right)-n_{2}\left[n_{1}\left(n_{1}-1\right) / 2-h_{1}\right]+n_{1} h_{2} \\
& =\frac{1}{2} n_{1} n_{2}\left(n_{1} n_{2}-n_{1}-n_{2}+1\right)+n_{2} h_{1}+n_{1} h_{2} .
\end{aligned}
$$

Suppose we have a curve $C^{n_{1} n_{2 n} n_{3}}$ which is the intersection of three varieties $V_{r_{1}}^{n_{1}}, V_{r_{2}}^{n_{2}}, V_{r_{3}}^{n_{3}}$ in $S_{r_{i}}$, where $r_{1}+r_{2}+r_{3}=2 r+1$. Let $h_{i}$ be the number of apparent double points on the curve $C^{n_{i}}$ in which an $S_{r-r_{i}+1}$ meets $V_{r_{i}}^{n_{i}}$. To find the number $h$ of apparent double points on $C^{n_{1} n_{2} n_{s}}$, we may reason as above or we may proceed as follows.

The curve $C^{n_{1} n_{2} n_{3}}$ may be considered as the intersection of $V_{r_{3}}^{n_{3}}$ and the variety $V_{r_{1}+r_{2}-r}^{n_{1} n_{2}}$, the latter being the intersection of $V_{r_{1}}^{n_{1}}$ and $V_{r_{2}}^{n_{2}}$. Let $h_{12}$ be the number of apparent double points on the curve $C^{n_{1} n_{2}}$ in which an $S_{r_{3}}$ meets $V_{r_{1}+r_{3}-r}^{n_{1} n_{2}}$ and its value is given by (3). Applying formula (3), we find, replacing $n_{1}, n_{2}, h_{1}, h_{2}$ by $n_{1} n_{2}, n_{3}, h_{12}, h_{3}$ respectively,

$$
h=\frac{1}{2} n_{1} n_{2} n_{3}\left(n_{1} n_{2} n_{3}-n_{1} n_{2}-n_{3}+1\right)+n_{3} h_{12}+n_{1} n_{2} h_{3} .
$$

Writing for $h_{12}$ its value from (3) in the above, we obtain

(4) $h=\frac{1}{2} n_{1} n_{2} n_{3}\left(n_{1} n_{2} n_{3}-n_{1}-n_{2}-n_{3}+2\right)$

$$
+n_{2} n_{3} h_{1}+n_{3} n_{1} h_{2}+n_{1} n_{2} h_{3}
$$

as the number of apparent double points on $C^{n_{1} n_{2} n_{3}}$.

Now let $q=4$. Then $C^{N}$, where $N=n_{1} n_{2} n_{3} n_{4}$, is the intersection of four varieties $V_{r_{1}}^{n_{1}}, V_{r_{2}}^{n_{2}}, V_{r_{3}}^{n_{3}}, V_{r_{4}}^{n_{3}}$, where $r_{1}+r_{2}+r_{3}+r_{4}=3 r+1$. We may regard $C^{N}$ as the intersection of $V_{r_{4}}^{n_{4}}$ and the variety $V_{r_{1}+r_{2}+r_{2}-2 r}^{n_{1} n_{2} n_{3}}$, the latter being the intersection of $V_{r_{1}}^{n_{1}}, V_{r_{2}}^{n_{2}}, V_{r_{3}}^{n_{3}}$, and 
apply (3) and (4), or we may regard it as the intersection of a $V_{r_{1}+r_{2}-r}^{n_{1} n_{2}}$ and a $V_{r_{3}+r_{4}-r}^{n_{3} n_{4}}$, the former being the intersection of $V_{r_{1}}^{n_{1}}$, $V_{r_{2}}^{n_{2}}$ and the latter that of $V_{r_{3}}^{n_{3}}, V_{r_{4}}^{n_{4}}$, and then apply (3) alone. Adopting the latter view, we have, replacing $n_{1}, n_{2}, h_{1}, h_{2}$ by $n_{1} n_{2}, n_{3} n_{4}, h_{12}, h_{34}$ respectively in (3),

$h=\frac{1}{2} n_{1} n_{2} n_{3} n_{4}\left(n_{1} n_{2} n_{3} n_{4}-n_{1} n_{2}-n_{3} n_{4}+1\right)+n_{1} n_{2} h_{34}+n_{3} n_{4} h_{12}$,

where $h_{12}$ and $h_{34}$ are the respective numbers of apparent double points on the curves $C^{n_{1} n_{2}}, C^{n_{3} n_{1}}$ in which an $S_{r_{3}+r_{4}-r}$ and an $S_{r_{1}+r_{2}-r}$ meet the varieties $V_{r_{1}+r_{2}-r}^{n_{1} n_{2}}$ and $V_{r_{3}+r_{4}-r}^{n_{3} n_{4}}$ respectively. Now $h_{12}$ is given by (3) and $h_{34}$ is also given by (3) if $n_{1}, n_{2}, h_{1}, h_{2}$ are replaced by $n_{3}, n_{4}, h_{3}, h_{4}$. Making these substitutions in the above, we have

(5) $h=\frac{1}{2} n_{1} n_{2} n_{3} n_{4}\left(n_{1} n_{2} n_{3} n_{4}-n_{1}-n_{2}-n_{3}-n_{4}+3\right)$

$$
+n_{2} n_{3} n_{4} h_{1}+n_{3} n_{4} n_{1} h_{2}+n_{4} n_{1} n_{2} h_{3}+n_{1} n_{2} n_{3} h_{4} \text {. }
$$

Without going through any further details we give at once the following formula, which can be easily verified, for the number of apparent double points on a curve $C^{N}$, where $N=n_{1} n_{2} \cdots n_{q}$ :

$$
\begin{aligned}
h= & \frac{1}{2} n_{1} n_{2} \cdots n_{q}\left(n_{1} n_{2} \cdots n_{q}-\sum n_{i}+q-1\right) \\
& +n_{1} n_{2} \cdots n_{q} \sum^{q} h_{i} / n_{i} .
\end{aligned}
$$

If $q=r-1$, we have, from (2), $r_{1}=r_{2}=\cdots=r_{r-1}=r-1$. Then the curve $C^{N}$ is the intersection of $r-1$ hypersurfaces. In this case, $h_{1}=h_{2}=\cdots=h_{r-1}=0$ as a plane section of a hypersurface cannot have apparent double points. Then (6) is reduced to (1).

As an illustration, let $C^{9}$ be the intersection of a $V_{3}^{3}$ and a $V_{3}^{\prime 3}$ in $S_{5}$. Since an $S_{3}$ in $S_{5}$ meets $V_{3}^{3}$ and $V_{3}^{\prime 3}$ each in a twisted cubic curve, we have $h_{1}=h_{2}=1$. We may use (3) or we may use (6) for $q=2$. Putting $n_{1}=n_{2}=3$, we have $h=24$ as the number of apparent double points on the curve $C^{9}$.

UNIVERSITY OF CALIForNia 